\title{
Study of the Morphology of Erythrocytes in Patients with Cervical Cancer: A Technique for Machine Recognition of the Shapes and Sizes of Erythrocytes SEM Images
}

\author{
S.N. Mamaeva ${ }^{1 *}$, O.A. Semenov ${ }^{1}$, I.V. Kononova ${ }^{2}$, A.R. Ermolaev ${ }^{1,3}$, V.V. \\ Gerasimova $^{1,3}$ and G.V· Maksimov ${ }^{4,5}$ \\ ${ }^{1}$ Ammosov North-East Federal University, Yakutsk, Russia \\ ${ }^{2}$ Yakut scientific center of complex medical problems, Yakutsk, Russia \\ ${ }^{3}$ State Budgetary Institution of the Republic of Sakha (Yakutia) Blood Transfusion Station, Yakutsk, Russia \\ ${ }^{4}$ Lomonosov Moscow State University (Moscow, Russia) \\ ${ }^{5}$ National Research Technological University MISiS (Moscow, Russia) \\ *Corresponding author. Email: sn.mamaeva@s-vfu.ru
}

\begin{abstract}
The aim of the study is to develop a technique for machine recognition of the shapes and sizes of scanning electron microscope (SEM) images of erythrocytes of patients with cervical cancer (CC) obtained on a SEM during radiation therapy (RT). The article considers the study of the morphology of erythrocytes of patients with CC who were underwent RT using machine image recognition methods based on images of erythrocytes obtained by SEM. It is shown that the use of this method already at the beginning of RT allows us to observe significant changes in the morphology of red blood cells of patients with cervical cancer, while the use the method of JMicroVision v1.2.7 states a slight difference of the diameters of red blood cells in the normal and pathological on the basis of the same experimental data. This paper presents for the first time a method for studying the morphology of erythrocytes in patients with cancer based on SEM images of erythrocytes. The presented automated program can recognize erythrocytes, calculate their number, diameters, areas, taking into account changes in morphology both in normal and pathological conditions, can work with a large amount of data with a significant acceleration of the calculation of parameters and with a greater approximation of these data to real ones, which significantly increases the efficiency of analysis in research, in the diagnosis and monitoring of therapy for CC and other types of diseases. In addition, these developments can help to increase the scope of scanning electron microscopes for solving a wide range of problems in medicine related to images in the field of diagnostics - medical introscopy.
\end{abstract}

Keywords: erythrocytes, cervical cancer, machine recognition technique, scanning electron microscope, SEM

\section{INTRODUCTION}

Cervical cancer (CC) is the fourth most common cancer among women in the world. The incidence of CC is higher in Africa, Asia, and Latin America. In the Russian Federation, morbidity levels are registered above the global average. Thus, the age-standardized incidence rate for 2018 was 6.2 per 100 thousand of the population. The increase in morbidity from 2007 to 2017 was $4.5 \%$ [1].

The annual increase in the number of patients with $\mathrm{CC}$ requires the development of new research, diagnostics and monitoring methods of the effectiveness 
of therapy. Despite the fact that the morphology of erythrocytes depends on a large number of parameters, currently the study of the shapes and sizes of red blood cells is an important point in the study of the cellular mechanisms of pathologies and in their diagnosis. In this regard, the use of a modern scanning electron microscope (resolution of $0.4 \mathrm{~nm}$ ) will allow to obtain a more detailed image of red blood cells compared to images obtained using an optical microscope (resolution of no more than 0.2 microns). Previously, images of the surface of erythrocytes were obtained at an increase of 100,000 times using a JEOL JSM-7800F SEM with a thermal field cathode of Schottky and with a Gentle Beam system at low accelerating voltages (1-2 kV) [2]. Increases of 1000 times in the work [3] were used to obtain statistical data on the morphology of erythrocytes of patients with CC during RT. The possibility of achieving this increase in magnification for SEM is easily realized and the images of erythrocytes are more informative than the images obtained on an optical microscope at the same magnification.

In [4], the problem of counting erythrocytes on images of blood smears obtained using an optical microscope is considered. The authors have developed a method of image segmentation, which is based on the detection of boundaries by the Canny method with the identification of circles in the image using the Hough transform. We used the refinement of the segmentation results based on the binary mask of objects and the background. The proposed algorithm made it possible to perform segmentation and counting of blood cells with an accuracy of up to $86 \%$.

The paper [5] considers the problem of making a diagnosis by hematological analysis of a digital image of red blood cells based on images of stained blood smears obtained using an optical microscope. The authors of the article describe methods of image preprocessing to reduce noise and the accuracy of segmentation of cell objects into classes. In conclusion, histograms of the distribution of the areas of cells Price-Jones curves - are constructed. To perform data analysis, a program in Python 2.7 with the OpenCV and Seaborn libraries was used.

It is obvious that the information content of the analysis of the obtained images depends not only on their quality and the resolution of the microscope, but also on the methods of their computer processing. With the development of computer technologies, it became possible to automate the processing of experimental data arrays with small time costs and with improved methods of their analysis. The creation of programs for processing the results of the study by the SEM method of blood smears of patients with CC is an urgent task, the solution of which can allow analyzing the causes of the disease at a new level, as well as monitoring the effectiveness of radiation therapy at the cellular level.
The purpose of this work is to develop a technique for machine recognition of the shapes and sizes of SEM images of erythrocytes of patients with cervical cancer during radiation therapy.

\section{MATERIALS AND METHODS}

The object of the study. The study used smears of venous blood containing K3-EDTA from 12 patients aged 45 to 55 years with cervical cancer. Tests were taken at the beginning of therapy and at each stage of the full course of treatment in 2 patients, and in the remaining 10 patients - only at the beginning of RT. Blood samples were prepared in the form of a dry, thin, even layer applied to a clean, fat-free slide for the study on SEM. SEM was used to study the morphology and surface of red blood cells in patients with $\mathrm{CC}$ at the beginning, after the first and second (final) stages of RT (remote and contact, respectively).

Radiation Treatment (RT). In the Yakut Republican Oncology Dispensary (Yakutsk, Russia), patients with CC were treated with radiotherapy. RT consists of two stages: first, distance RT therapy using the Elekta Synergy accelerator (United Kingdom, external beam radiation therapy, 6-18 MeV); second, brachytherapy using the MultiSourse HDR device (Germany, brachytherapy with Cobalt-60 source). At the first stage, the treatment was performed on a linear accelerator in the mode of working with electrons with an energy of $6 \mathrm{MeV}$. The therapy was carried out as follows: three times for 5 days daily with a break of two days, i.e. at the first stage only 15 fractions of 2 Gy were carried out. Then, without a break between the first and second stage, contact radiation therapy of 5 Gy was performed, interspersed with distanced radiation therapy of $2 \mathrm{~Gy}$ of 5 fractions each type of radiation therapy. Blood samples were collected at the beginning, middle and end of the remote therapy, then in the middle and at the end of the contact radiotherapy. Multiple methods (3D CRT, IMRT, VMAT) were employed in the course of therapy to ensure precision delivery of high doses to the tumour and low doses to healthy tissue.

The study was carried out in compliance with Ethical Principles for Medical Research Involving Human Subjects, Adopted by the 18th WMA General Assembly, Helsinki, Finland, June 1964, and amended by the 52nd WMA General Assembly, Edinburgh, Scotland, October 2000. The study received approval of the Local Committee on Biomedical Ethics of Ammosov North-Eastern Federal University (protocol No. 13 of April 4, 2018, decision No. 2). All patients gave their written informed consent.

SEM imaging. We used a high-resolution SEM JSM-7800F (Japanese Electron-Optical Laboratory, JEOL, Japan), equipped with a thermal field cathode of Schottky and a super hybrid lens. The microscope is 
equipped with a Gentle Beam system, which reduces the electron velocity of the emission beam and allows obtaining sufficiently high-quality images of biological samples at low accelerating voltages. Using SEM, the relief of the surface of red blood cells in blood smears was studied without spraying conductive coatings and without damaging the object, which made it possible to identify nanoscale objects (resolution of $1.2 \mathrm{~nm}$ ): the magnification range is $1-100,000$ at a voltage of $1-2 \mathrm{kV}$. The measurements were made by detector of secondary electron. The accelerating voltage during the study of the samples was 1 or $1.1 \mathrm{kV}$, and the working distance (WD) varied in the range from 3.9 to $6.0 \mathrm{~mm}$.

Methods of machine recognition. The high-level programming language Python [6] was used using the libraries OpenCV [7], pytesseract [8], numpy [9], matplotlib [10] and Skimage [11] to process the experimental data. It is known that the SEM technology allows obtaining images only in shades of gray, depending on the density of the distribution of the detected secondary electrons, which often leads to the appearance of some artifacts over the entire surface of the object under study. To determine the diameters of red blood cells and their number, taking into account this feature of the SEM data, the following various computer programs were used: 1) the use of linear filters for image stabilization; 2) determination of the boundaries of objects (red blood cells) using the Canny operator [12]; 3) recognition of erythrocytes using the Hough method [13, 14]. The obtained data were analyzed by constructing dependencies, which are a graphical representation of the ratios of the values of the diameters of red blood cells to the percentage of erythrocytes (according to Price-Jones). Applying the functions of the segmentation algorithm for our program, we obtained images of pre-colored images of erythrocytes. To obtain extremums from the approximated curve of radius vectors, the function of image moments (the Ramer-Douglas-Pecker algorithm) was used, which is necessary for identifying the forms of red blood cells [15].

Several methods for calculating radii were used and two different methods for recognizing cells were applied to determine the linear dimensions of red blood cells. One of the methods of cell recognition is a procedure based on the calculation of the Hough transformation and allows you to accurately determine the diameter and contour of the area under study with a pre-set range of changes in the estimated minimum and maximum radii of objects. The second method is the application of a program that takes into account the loss of part of the boundaries of erythrocytes after applying the Canny operator.

\section{RESULTS AND THEIR DISCUSSION}

In the course of the study (SEM with an increase of 1,000 times), based on the identification of 128 erythrocytes, including $21.09 \%$ of microcytes, $50.0 \%$ of normocytes, $28.91 \%$ of macrocytes from their total number), the Price-Jones curve was obtained. Fig. 1 (a) and (b) show a SEM image of erythrocytes after applying the Hough method and the Price-Jones curve of a control group blood sample. The data obtained after calculating the diameters and the number of erythrocytes from the control group are characterized by the Price-Jones curve - the number of erythrocytes depending on the diameter (Fig. 1b). Note that there is a slight shift of the curve to the left side from the normal distribution.

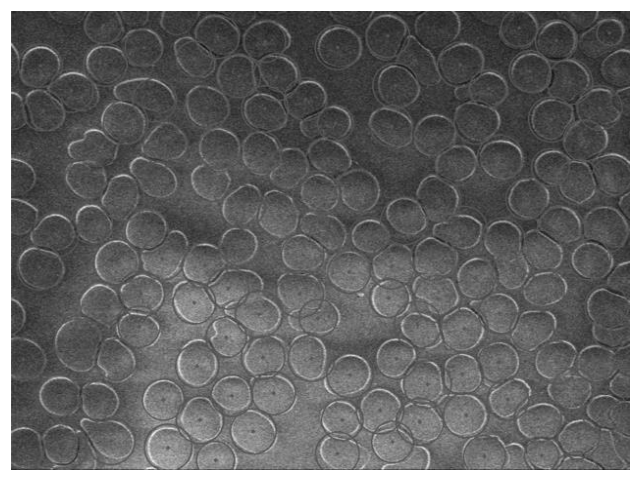

Figure 1a. SEM images of red blood cells after applying the Hough method

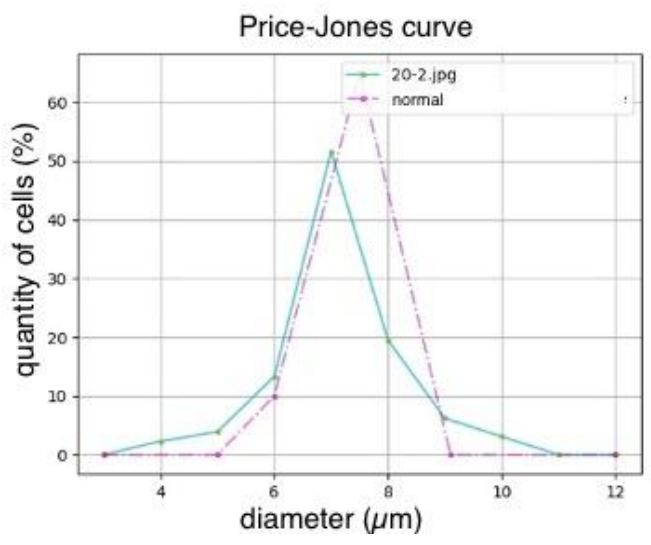

Figure 1b. The Price-Jones curve of a blood sample of the control group

When examining a blood sample in pathology, the following types of erythrocytes were detected in the image of erythrocytes with an increase of 1,000 times: microcytes - $56.72 \%$, normocytes - $31.34 \%$, macrocytes $-11.94 \%$, megacytes $-0 \%$.

In Fig. 2a a SEM image of erythrocytes and a PriceJones curve for erythrocytes are presented in case of pathology. In pathology, there is a significant shift in the 
distribution of the number of red blood cells and an expansion of the base of the graph relative to the normal distribution graph (Fig. 2b).

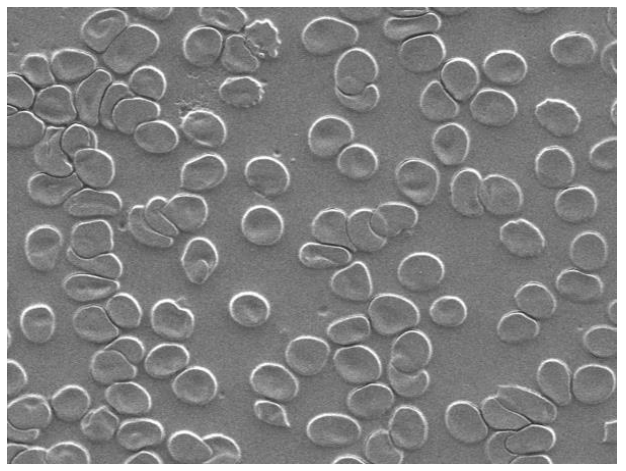

Figure 2a. SEM image of erythrocytes in pathology

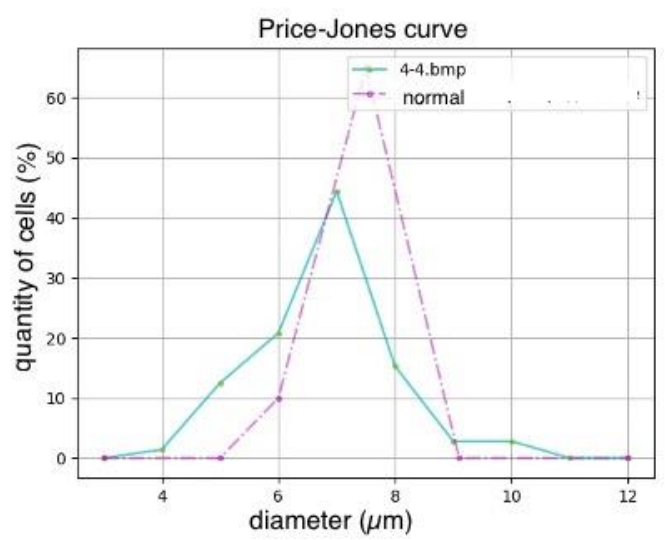

Figure 2b. The Price-Jones curve in pathology

The comparison of the Price-Jones curves indicates the differences between the norm and pathology and corresponds to the literature data obtained using the JMicroVision v1 program.2.7 [2].

Further, using the data of SEM images of blood samples of patients with CC (Fig. 3) and the control group and using this complex program, graphs of comparisons of the densities of the distribution of linear dimensions, as well as the surface areas of erythrocytes were obtained (Fig. 4).

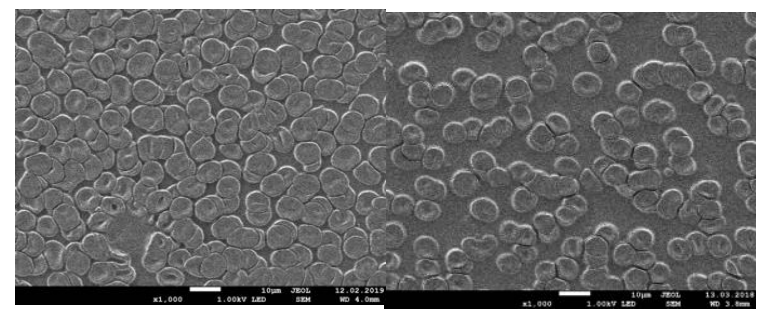

Figure 3. SEM images of erythrocytes of patients with $\mathrm{CC}$ before the start of RT at a magnification of 1,000 times
The results obtained indicate that there are differences in the parameters of the shapes and sizes of erythrocytes in patients with CC compared with the samples of the control group. A comparison of the parameters of the distribution of diameters and surface area of erythrocytes shows that before RT, these parameters are less in patients with $\mathrm{CC}$ than in the control group, taking into account their altered forms.

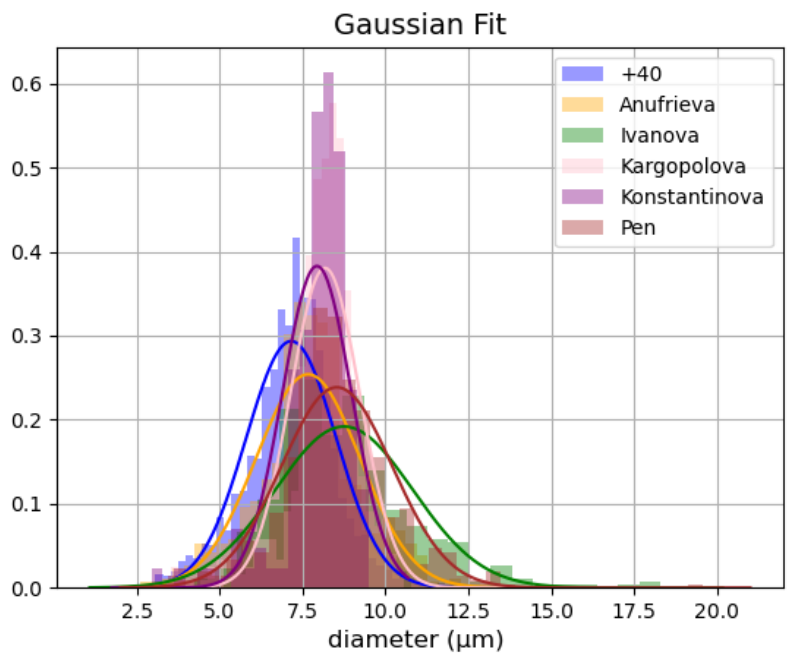

Figure 4a. Gaussian distributions the density of the distribution of the diameters of erythrocytes in normal and pathological conditions

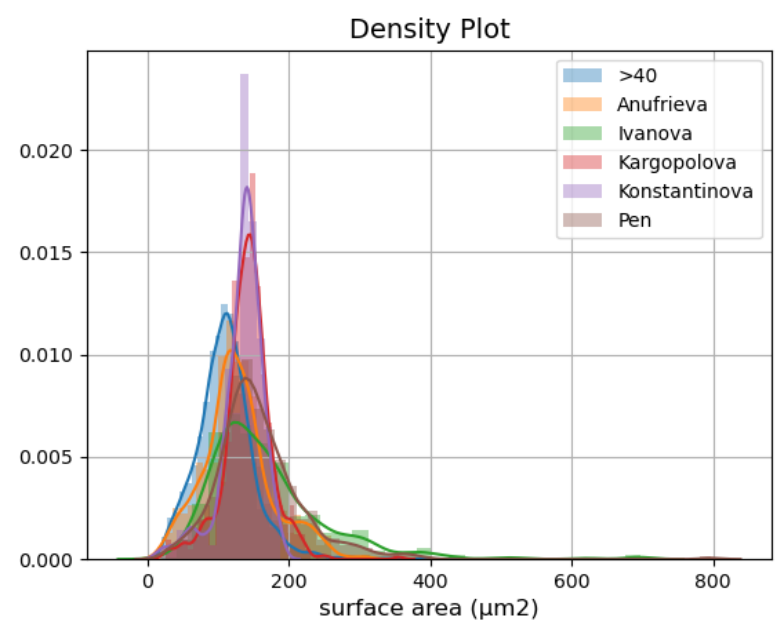

Figure 4b. Gaussian distributions: the density of the distribution of the surface area of erythrocytes in normal and pathological conditions before RT

Earlier, using SEM studies [2, 3], an increase in the number of dysmorphic erythrocytes was detected during RT, which was poorly studied using conventional blood analyzers, optical microscopy due to low resolution and lack of data analysis using machine learning (for example, using JMicroVision v1.2.7). And the use of this method of machine image recognition already at the beginning of RT allows us to observe changes in the morphology of erythrocytes of patients with CC (Fig. 3, 4), 
while the use of JMicroVision v1.2.7 on the basis of experimental data on SEM, it states a slight difference in the diameters of red blood cells, for example, before $\mathrm{RT}$ in patients with CC [2].

It was previously assumed that changes in the morphology of erythrocytes during RT are due to the effect of ionizing radiation on the body [3]. And in accordance with this, it was concluded that further studies of the morphology of erythrocytes during RT are necessary to determine the effectiveness of RT. Numerical values of the average diameters and surface areas of erythrocytes using a machine program for recognizing erythrocytes images were obtained with less time, with greater approximations to the real morphological parameters of red blood cells.

\section{CONCLUSIONS}

So, the proposed processing of the results and their analysis using a computer image recognition program can be effectively used in the analysis of images of erythrocytes obtained by scanning electron microscopy. The presented automated program can recognize erythrocytes, calculate their number, diameters, areas, taking into account changes in morphology both in normal and in pathology. It is important that the obtained data is stored in a text format and can be used when plotting the Price-Jones curve. It should also be noted that the program can work with a large amount of data with a significant acceleration of the calculation of parameters and with a greater approximation of these data to real ones, which significantly increases the efficiency of analysis in research, in the diagnosis and monitoring of therapy for cervical cancer and other types of diseases.

In addition, these developments can help to increase the scope of scanning electron microscopes, for example, in their application to solve a wide range of problems in medicine related to images in the field of diagnostics - medical introscopy.

\section{REFERENCES}

[1] A.D. Kaprin, V.V. Starinskiy, G.V. Petrova, P. Herzen, Mos. Oncol. Res. Inst. 250 (2019).

[2] S.N. Mamaeva, G.V. Maksimov, S.R. Antonov et al., AIP Conf. Proc., vol. 2041, 2018, p. 1.

[3] S.N. Mamaeva, I.V. Kononova, M. Ruzhansky et al., IJB 10(1) (2020) 70-75.

[4] A.V. Dyrnaev, A.S. Potapov, Sci. tech. b. inf. tech., mechan. and optics 1 (2012) 77.

[5] E.S. Soinikova, D.S. Batishchev, V.M. Mikhelev, Inf. tech. 3 (2018) 3.

[6] https://www.python.org
[7] https://opencv.org

[8] https://github.com/tesseract-ocr/tesseract

[9] https://numpy.org

[10] https://matplotlib.org

[11] https://scikit-image.org/docs/dev/api/skimage.html

[12] https://www.jeol.co.jp/en/

[13] J. Canny, IEEE 6 (1986) 679-698.

[14] M.A. Kudrina, B. S. Sci. C. Rus. A. Sci. 16 (2014) $4-2$. 